Journal for ImmunoTherapy of Cancer

\title{
Incidence of thromboembolism in patients with melanoma on immune checkpoint inhibitor therapy and its adverse association with survival
}

\author{
Tamara A Sussman (D) , ${ }^{1}$ Hong Li, ${ }^{1}$ Brian Hobbs, ${ }^{2}$ Pauline Funchain, ${ }^{1}$ \\ Keith R McCrae, ${ }^{1}$ Alok A Khorana ${ }^{1}$
}

To cite: Sussman TA, Li H, Hobbs B, et al. Incidence of thromboembolism in patients with melanoma on immune checkpoint inhibitor therapy and its adverse association with survival. Journal for ImmunoTherapy of Cancer 2021;9:e001719. doi:10.1136/ jitc-2020-001719

Abstract previously presented as poster at ASCO-SITC 2020 annual meeting. Abstract presented as poster at SITC 2020 annual meeting.

Accepted 11 December 2020

Check for updates

(c) Author(s) (or their employer(s)) 2021. Re-use permitted under CC BY-NC. No commercial re-use. See rights and permissions. Published by BMJ.

${ }^{1}$ Department of Hematology and Oncology, Taussig Cancer Institute, Cleveland Clinic Foundation, Cleveland, Ohio, USA

${ }^{2}$ Department of Population Health, Dell Medical School, The University of Texas at Austin, Austin, Texas, USA

Correspondence to

Dr Alok A Khorana;

khorana@ccf.org

\section{ABSTRACT}

Background Thromboembolism (TE) in cancer significantly contributes to morbidity and mortality. Little is known about the incidence of arterial TE (ATE) and venous TE (VTE) in patients with melanoma on immune checkpoint inhibitor (ICI) therapy.

Methods We conducted a retrospective cohort study of patients with melanoma receiving ICI from July 2015 through December 2017 at the Cleveland Clinic. TE, including VTE events of deep venous thrombosis, pulmonary embolism, visceral vein thrombosis, and ATE events of myocardial infarction, stroke, peripheral arterial embolism, or transient ischemic attack after ICl initiation were identified. Overall survival (OS) from ICI initiation was estimated by Kaplan-Meier and Cox hazard models; associations between TE, ICl regimen, and clinical risk factors were evaluated using log-rank test.

Results The study population comprised 228 patients with median age of 65 years (23-91 years), $67 \%$ male, and median follow-up of 27.3 months. Pembrolizumab was most commonly used (38.7\%), followed by combination of ipilimumab plus nivolumab (29.4\%), ipilimumab (20\%), and nivolumab (12.3\%). Most had stage IV disease $(81.1 \%)$ and $11 \%$ had brain metastases (BM) at treatment initiation. Fifty-one TE events occurred in 47 patients (20.6\%), including 37 (16.2\%) VTE and 14 (6.1\%) ATE. Cumulative incidence of TE after ICI initiation was 9.3\% (95\% Cl: $6.0 \%$ to $13.6 \%$ ) at 6 months, and $16.0 \%(95 \%$ Cl: $11.6 \%$ to $21.2 \%$ ) at 12 months. The 6 -month and 12-month VTE cumulative incidence rates were higher with combination ICl than single agent ( $16.7 \%$ vs $5.0 \%$ and $21.3 \%$ vs $9.5 \%$, respectively; $p=0.02$ ). Risk factors significantly associated with VTE in multivariate analysis included combination ICI (HR 2.70; $95 \%$ Cl: 1.28 to 5.70; $\mathrm{p}=0.009$ ), Khorana Score $\geq 1$ (HR 2.24; $95 \%$ Cl: 1.06 to $4.74 ; p=0.03$ ), history of coronary artery disease (HR 2.71; $95 \% \mathrm{Cl}: 1.16$ to 6.29$) ; \mathrm{p}=0.02)$, and anticoagulation at treatment start (HR 4.14; 95\% Cl: 1.60 to $10.7 ; p=0.003$ ). Of patients without $\mathrm{BM}, \mathrm{OS}$ was worse in patients with TE compared with those without (2-year OS $50.8 \%$ vs $71.3 \%$; HR 2.27; $95 \%$ Cl: 1.36 to $3.79 ; p=0.002$ ), when adjusted for age and stage.

Conclusions ICl is associated with a high incidence of $\mathrm{TE}$ in patients with melanoma, with higher rates with combination therapy; TE is associated with substantial worsening of survival. Further studies are needed to identify pathophysiology, biomarkers, and preventive approaches.

\section{INTRODUCTION}

The risk of venous thromboembolism (VTE) is increased in patients with cancer, by at least fourfold-sevenfold over the general population. ${ }^{12}$ VTE significantly contributes to morbidity and a worse overall prognosis in patients with cancer. ${ }^{3}$ VTE rates ranging from $2 \%$ to $14 \%$ have been reported in different populations of patients with cancer, with the highest VTE risk identified in patients with pancreatic $(\sim 11 \%)$, lung $(\sim 8 \%)$, and stomach cancer $(\sim 8 \%) .{ }^{1-4}$ Similarly, rates of arterial thromboembolism (ATE) and mortality are increased in patients with cancer. Both VTE and ATE have been associated with worse prognosis across several malignancies. ${ }^{56}$ VTE rates are known to increase with the use of specific chemotherapy and targeted therapy agents. ${ }^{7-11}$ However, limited data are available regarding the incidence of VTE and ATE for patients with melanoma, with one cohort suggesting a VTE rate of $2.6 \% .{ }^{12}$ A smaller study of melanoma patients $(n=95)$ suggests a VTE rate of $25 \%$, with the majority of VTE developing while on chemotherapy. ${ }^{13}$

With the advent of immune checkpoint inhibitor (ICI) therapy in melanoma in 2011, the treatment landscape has revolutionized, with improved survival and outcomes. However, little is known about neither the incidence of TE nor the extent to which its occurrence impacts the therapeutic efficacy of ICI for melanoma patients. A recent study reports ATE rate of $4.9 \%(6 / 122)$ and VTE rate of $4.1 \%(5 / 122)$ in patients with lung, stomach, or kidney cancer receiving ICI. ${ }^{14}$ Three ICIs are approved by the Food and Drug Administration in melanoma, including ipilimumab (approved in 2011), nivolumab 
(2014), and pembrolizumab (2015). These agents can be given as monotherapy, or ipilimumab can be given in combination with nivolumab. Combination therapy in melanoma has been associated with higher rates of immune-related adverse events like rash, arthralgia, colitis, and endocrinopathy, when compared with monotherapy. ${ }^{15}$ However, the thromboembolic risk added by immunotherapy in ICI-treated melanoma patients has yet to be evaluated.

A validated risk model for VTE, also known as the Khorana Score, has been developed based on data from a large, prospective US national cohort study of patients initiating a new chemotherapy regimen for certain solid tumors and lymphoma. ${ }^{7}$ This risk model has been validated by independent investigators across a range of solid tumors, ${ }^{8916}$ and has been incorporated into clinical guidelines for VTE risk assessment. ${ }^{17}{ }^{18}$ However, the model's reliability for predicting VTE in melanoma patients receiving ICI therapy has not been established.

In this study, we aim to assess the incidence of VTE and ATE in patients with melanoma receiving ICI, interrogate its potential association with therapy regimens and clinical risk factors, identify the predictive benefit of a previously validated VTE risk score, and ascertain its association with survival.

\section{METHODS}

We conducted a retrospective cohort study of patients with melanoma receiving ICI therapy, including ipilimumab, nivolumab, or pembrolizumab from July 2015 through December 2017 at the Cleveland Clinic Taussig Cancer Institute and its satellite oncology clinic locations. TE, including VTE events and ATE events, after initiation of ICI therapy was identified by manual chart review of the electronic medical record, including review of radiographic reports (CT scans of the head/the chest/the abdomen or lower extremity ultrasound) and provider documentation. VTE was defined as deep venous thrombosis (DVT), pulmonary embolism (PE), and visceral vein thrombosis. ATE was defined as myocardial infarction (MI), stroke, peripheral arterial embolism, or transient ischemic attack (TIA). Hospitalization was defined as greater than or equal to one night stay in the hospital. History of VTE and ATE was defined as VTE and ATE before ICI therapy initiation. History of coronary artery disease $(\mathrm{CAD})$ was defined as angina or acute coronary syndrome before ICI therapy initiation. Anticoagulation therapy at treatment start was defined by therapeutic or prophylactic anticoagulation prescribed by a provider with one of the following agents: apixaban, dabigatran, edoxaban, enoxaparin, heparin, rivaroxaban, or warfarin. Khorana Score for VTE risk was calculated by addition of one point for each of the following: pre-treatment platelet count $\geq 350 \times 10^{9} / \mathrm{L}$, hemoglobin level $<10 \mathrm{~g} / \mathrm{dL}$ or using red blood cells (RBC) growth factors, pre-treatment leukocyte count $>11 \times 10^{9} / \mathrm{L}$, and body mass index (BMI) $\geq 35 \mathrm{~kg} / \mathrm{m}^{2}$. All patients were given zero points for cancer type. Patients less than 18 years of age were excluded $(\mathrm{N}=1)$. The study was approved by the institutional review board.

\section{Statistical analysis}

Statistical analyses were devised to evaluate the time from ICI initiation to first TE event (VTE and ATE). The majority of VTE and ATE events were observed within 12 months of initiation of ICI therapy. Associations of ICI with 12-month VTE-free survival and 12-month ATE-free survival were evaluated. Patients who had TE after 12 months or who did not have TE but had follow-up for more than 12 months were censored at 12 months.

Demographics, ICI regimen, and other clinical factors at time of ICI initiation were described descriptively using median (IQR) and frequency (percentage). Clinical prognostic factors hypothesized to impact VTE or ATE were explored initially using Kaplan-Meier method. Univariate Cox proportional hazards regression estimated associations with TE for each individual factor. Death before TE was observed in a subset of patients. Regression analysis used the method of Fine and Gray to model death as a competing event. ${ }^{19}$ Factors exhibiting association with VTE or ATE $(p<0.10)$ in univariate Cox competing risk analyses were further examined with multiple Cox competing risk analyses to evaluate their conjoint, independent effects. A final model was selected using Akaike information criterion. ${ }^{20}$ Patients on anticoagulation with or without prior DVT at treatment start were included in the main VTE-free survival analysis for the new event. Analysis excluding patients on anticoagulation with prior DVT at time of treatment was also performed. In addition, overall survival (OS) from start of ICI was compared between patients with and without TE using Kaplan-Meier method and Cox hazard model. Due to the interaction between TE and brain metastasis on OS, comparisons of OS were stratified by brain metastasis (the presence/the absence). Finally, characteristics of TE and other outcomes were described descriptively. All analyses were performed using SAS V.9.4. Two-sided $p$ values are presented in tables and figures, $\mathrm{p}<0.05$ is considered as statistically significant.

\section{RESULTS \\ Patient characteristics}

The study population comprised 228 patients with a median age of 65.5 years (range: 23-91 years) (table 1 ). Patients were predominantly white $(98.2 \%)$ males $(67.5 \%)$ with median follow-up time of 27.3 months (IQR: 9.3-38.9 months) from ICI therapy initiation. Most patients had stage IV disease $(\mathrm{n}=181,81.1 \%)$, and $11 \%(\mathrm{n}=25)$ had brain metastases at treatment initiation. Overall, $8.8 \%(\mathrm{n}=20)$ of patients had prior history of VTE, and $12.7 \%(n=29)$ had prior history of CAD. Of 228 patients, $4.8 \%(\mathrm{n}=11)$ were on anticoagulation at treatment initiation, including 10 patients on therapeutic anticoagulation and 1 on prophylactic anticoagulation. 
Table 1 Patient characteristics

\begin{tabular}{|c|c|}
\hline & \multirow{2}{*}{$\begin{array}{l}\text { All patients } \\
\mathrm{N}=228(\%)\end{array}$} \\
\hline & \\
\hline Median age at diagnosis (range) (years) & $65.5(23-91)$ \\
\hline \multicolumn{2}{|l|}{ Gender } \\
\hline Male & $154(67.5)$ \\
\hline Female & $74(32.5)$ \\
\hline \multicolumn{2}{|l|}{ Race } \\
\hline White & $224(98.2)$ \\
\hline Black or African American & $4(1.8)$ \\
\hline \multicolumn{2}{|l|}{ Stage } \\
\hline III & $43(18.9)$ \\
\hline IV & $185(81.1)$ \\
\hline Brain metastases at treatment start & $25(11.0)$ \\
\hline History of VTE & $20(8.8)$ \\
\hline History of ATE & $9(3.9)$ \\
\hline History of CAD & $29(12.7)$ \\
\hline $\begin{array}{l}\text { Anticoagulation with/without prior DVT at } \\
\text { treatment start }\end{array}$ & $11(4.8)$ \\
\hline \multicolumn{2}{|l|}{ Khorana Score at treatment start } \\
\hline 0 & $154(67.5)$ \\
\hline 1 & $50(22.0)$ \\
\hline 2 & $11(4.8)$ \\
\hline 3 & $8(3.5)$ \\
\hline Unknown & $5(2.2)$ \\
\hline \multicolumn{2}{|l|}{ ICl type(s) } \\
\hline Ipilimumab & $46(20.2)$ \\
\hline Ipilimumab/nivolumab & $67(29.4)$ \\
\hline Nivolumab & $28(12.3)$ \\
\hline Pembrolizumab & $87(38.1)$ \\
\hline
\end{tabular}

ATE, arterial thromboembolism; CAD, coronary artery disease; DVT, deep venous thrombosis; VTE, venous thromboembolism.

Of these 11 patients on anticoagulation, $73 \% \quad(n=8)$ received anticoagulation for history of VTE and $27 \%$ $(n=3)$ received anticoagulation for atrial fibrillation. The majority of patients had a Khorana Score of 0 at treatment initiation $(\mathrm{n}=154,67.5 \%), 22 \%(\mathrm{n}=50)$ had a score of 1 , and $8.3 \%(\mathrm{n}=19)$ had a score of 2-3. Pembrolizumab was the most commonly used agent $(n=87,38.7 \%)$, followed by combination of ipilimumab plus nivolumab $(n=67$, $29.4 \%)$, ipilimumab $(n=46,20 \%)$, and nivolumab $(n=28$, $12.3 \%)$.

\section{TE events}

Fifty-one TE events occurred in 47 patients (20.6\%), of which $37(16.2 \%)$ were VTE and $14(6.1 \%)$ were ATE (table 2). Of VTE events, DVT comprised 17 (46.0\%), PE comprised 9 (24.3\%), DVT with PE comprised 9 (21.6\%), visceral vein thrombosis comprised $2(5.4 \%)$, and 1 had DVT with visceral vein thrombosis $(2.7 \%)$. Of patients
Table 2 Characteristics of thromboembolic events after ICI initiation

\begin{tabular}{|c|c|}
\hline & $\mathbf{N}(\%)$ \\
\hline VTE & $37(16.2)$ \\
\hline Deep vein thrombosis & $17(46.0)$ \\
\hline PE & $9(24.3)$ \\
\hline Visceral vein thrombosis & $2(5.4)$ \\
\hline Deep vein thrombosis+PE & $8(21.6)$ \\
\hline $\begin{array}{l}\text { Deep vein thrombosis+visceral vein } \\
\text { thrombosis }\end{array}$ & $1(2.7)$ \\
\hline \multicolumn{2}{|l|}{ ICl type(s) } \\
\hline Ipilimumab & 7 (18.9) \\
\hline Ipilimumab/nivolumab & $16(43.2)$ \\
\hline Nivolumab & $3(8.1)$ \\
\hline Pembrolizumab & $11(29.7)$ \\
\hline Hospitalized for VTE & $23(62.2)$ \\
\hline Hospitalized within 30 days before VTE & $9(24.3)$ \\
\hline $\mathrm{ICl}$ stopped after VTE & $9(24.3)$ \\
\hline $\mathrm{ICI}$ delayed after VTE & $5(13.5)$ \\
\hline Death as result of VTE & $1(2.7)$ \\
\hline ATE & $14(6.1)$ \\
\hline MI & $5(35.7)$ \\
\hline Stroke & $8(57.2)$ \\
\hline TIA & $1(7.1)$ \\
\hline \multicolumn{2}{|l|}{ ICl type(s) } \\
\hline Ipilimumab & $3(21.4)$ \\
\hline Ipilimumab/nivolumab & $3(21.4)$ \\
\hline Nivolumab & $1(7.1)$ \\
\hline Pembrolizumab & $7(50.0)$ \\
\hline Hospitalized for ATE & $11(78.6)$ \\
\hline Hospitalized within 30 days before ATE & $5(35.7)$ \\
\hline $\mathrm{ICl}$ stopped after ATE & $4(28.6)$ \\
\hline ICI delayed after ATE & $2(14.3)$ \\
\hline Death as result of ATE & $6(43.0)$ \\
\hline
\end{tabular}

ATE, arterial thromboembolism; $\mathrm{ICI}$, immune checkpoint inhibitor; MI, myocardial infarction; PE, pulmonary embolism; TIA, transient ischemic attack; VTE, venous thromboembolism.

on anticoagulation with or without history of DVT at treatment start, $5(45.5 \%)$ developed new VTE. Of ATE events, stroke comprised $9(57.2 \%)$, MI comprised 5 $(35.7 \%)$, and 1 had TIA $(7.1 \%)$. Twenty-three patients $(62.2 \%)$ were hospitalized for VTE, while $11(78.6 \%)$ were hospitalized for ATE. ICI was permanently discontinued in 9 patients $(24.3 \%)$ with VTE and 4 patients $(28.6 \%)$ with ATE. Treatment was delayed in 5 patients with VTE (13.5\%) and 2 with ATE (14.3\%), with mean treatment delay of 61 days with either event.

The majority of VTE $(n=29,78.4 \%)$ and ATE $(n=10$, $71.4 \%$ ) occurred within 12 months of ICI initiation. Of 
A: TE incidence

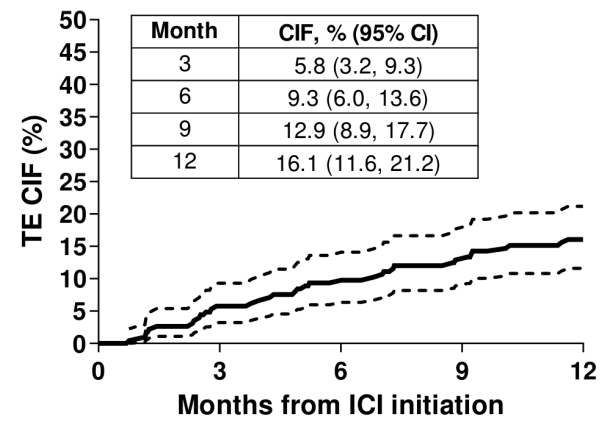

B: VTE incidence

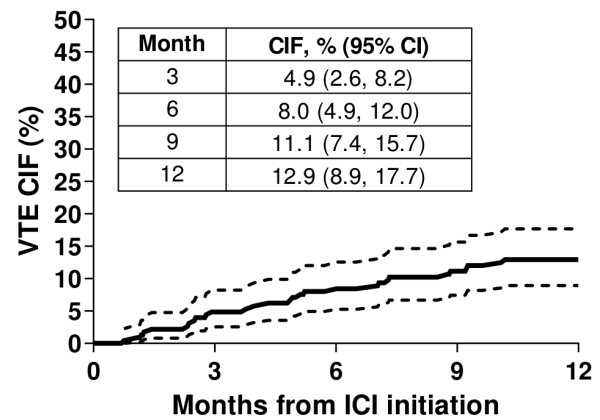

C: ATE incidence

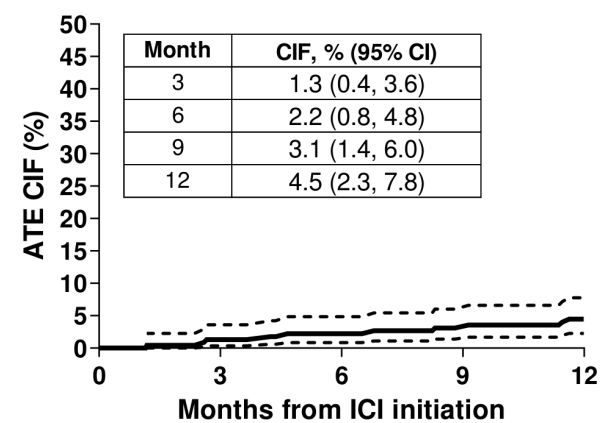

Figure 1 Cumulative incidence function graphs of (A) TE at 3 months, 6 months, 9 months, and 12 months of $\mathrm{ICl}$ initiation with 95\% Cls; (B) VTE at 3 months, 6 months, 9 months, and 12 months of $\mathrm{ICl}$ initiation with $95 \% \mathrm{Cls}$; and (C) ATE at 3 months, 6 months, 9 months, and 12 months of $\mathrm{ICl}$ initiation with $95 \% \mathrm{Cls}$. ATE, arterial thromboembolism; CIF, cumulative incidence function; $\mathrm{ICl}$, immune checkpoint inhibitor; TE, thromboembolism; VTE, venous thromboembolism.

those with VTE at 12 months, 14 (48.3\%) were receiving combination of ipilimumab/nivolumab therapy and $15(21.7 \%)$ were receiving single agent ICI. Of those with ATE at 12 months, 2 (20\%) were receiving combination ICI and $8(80 \%)$ single agent ICI. The 6-month and 1-year cumulative incidence rates of TE were $9.3 \%$ (95\% CI: $6.0 \%$ to $13.6 \%$ ) and $16.0 \%$ (95\% CI: $11.6 \%$ to $21.2 \%$ ), respectively. The 6 -month and 1 -year cumulative incidence rates of VTE were $8.0 \%$ (95\% CI: $4.9 \%$ to $12.0 \%$ ) and $12.9 \%$ (95\% CI: $8.9 \%$ to $17.7 \%$ ), respectively. The 6-month and 1-year cumulative incidence rates of ATE were $2.2 \%$ (95\% CI: $0.84 \%$ to $4.8 \%)$ and $4.5 \%$ (95\% CI: $2.3 \%$ to $7.8 \%$ ), respectively (figure 1 ).
A: Cumulative incidence of VTE stratified by ICI regimen

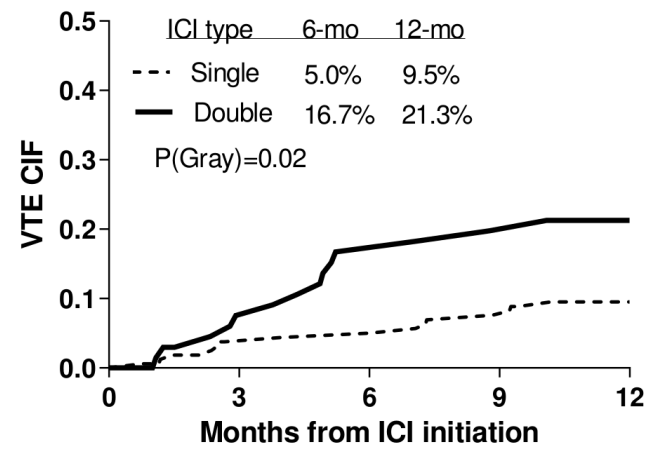

B: Cumulative incidence of VTE stratified by Khorana score

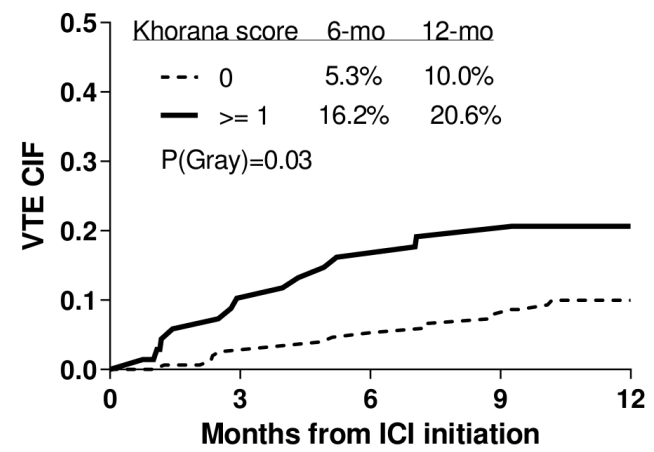

C: Cumulative incidence of VTE stratified by CAD history

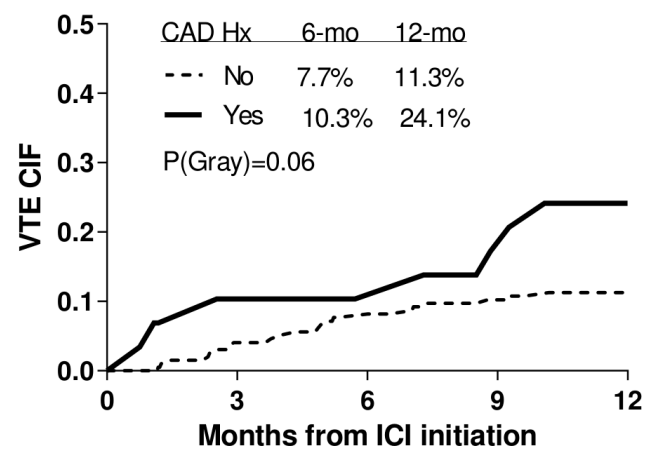

Figure 2 Cumulative incidence of VTE using Fine and Gray method with death as a competing risk for factors associated with VTE in univariate analysis $(A)$ stratified by $\mathrm{ICl}$ regimen of either single or doublet $I C I$ therapy $(p=0.02) ;(B)$ stratified by Khorana Score of 0 or $\geq 1$ at treatment initiation $(p=0.03)$; and $(C)$ stratified by the presence or the absence of CAD history $(p=0.06)$. CAD, coronary artery disease; CIF, cumulative incidence function; $\mathrm{ICl}$, immune checkpoint inhibitor; mo, months; TE, thromboembolism; VTE, venous thromboembolism.

The 6-month and 1-year cumulative VTE incidence rates were higher in patients receiving combination ICI as compared with those receiving single agent ICI $(16.7 \%$ vs $5.0 \%$ at 6 months and $21.3 \%$ vs $9.5 \%$ at 12 months, respectively; $\mathrm{p}=0.02$ ) (figure 2 ). The 6-month and 1-year cumulative ATE incidence rates were similar in patients receiving combination or single agent ICI, respectively (2.5\% vs $1.5 \%$ at 6 months and $5.1 \%$ vs $3.0 \%$ at 12 months; $\mathrm{p}=0.50)$. 
Table 3 Univariable and multivariable associations between clinical factors and VTE

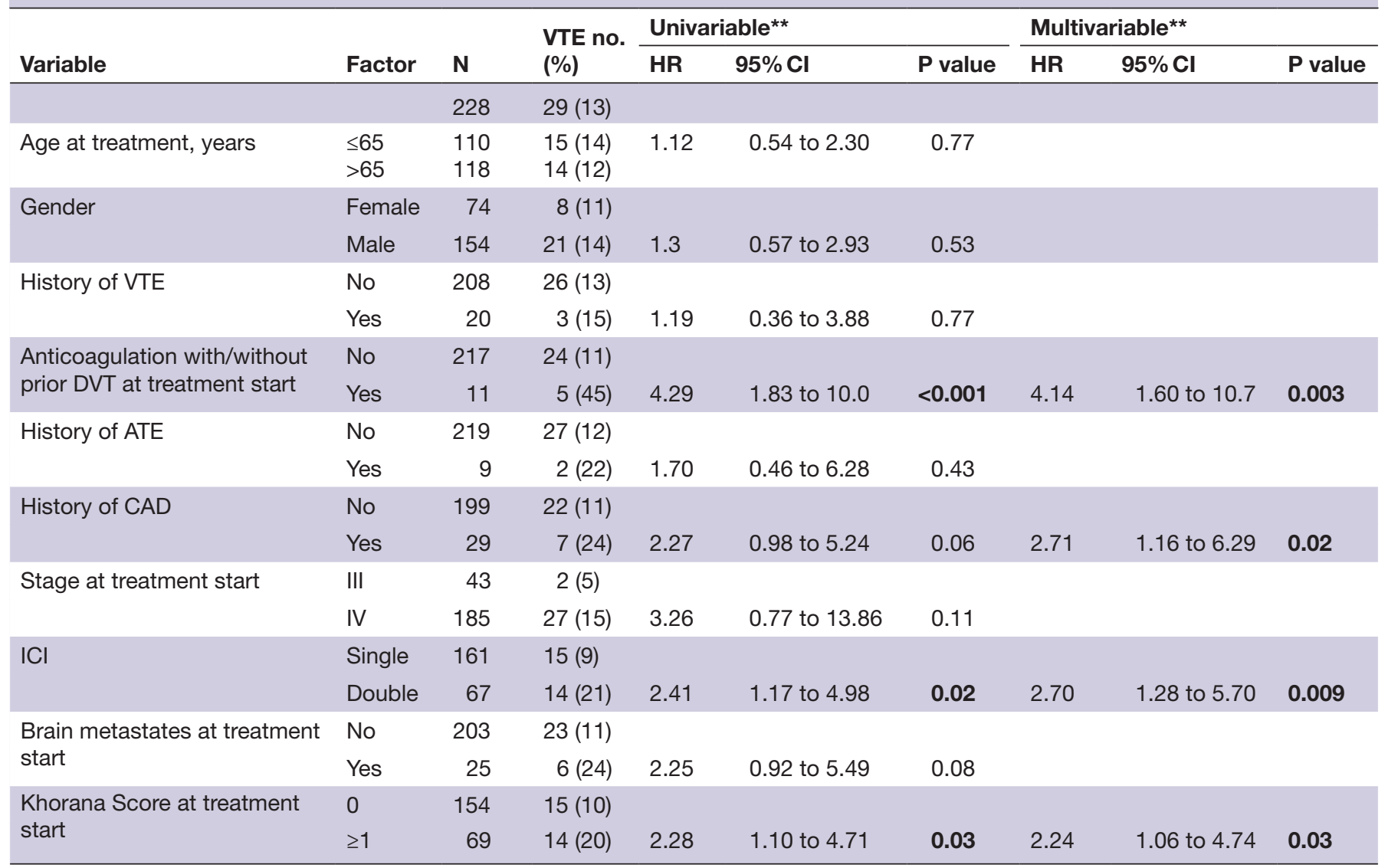

*Univariable and multivariable Cox proportional hazard analysis calculated with death as competing risk. Bold type indicates $\mathrm{p}$ values $<0.05$.

ATE, arterial thromboembolism; CAD, coronary artery disease; DVT, deep venous thrombosis; VTE, venous thromboembolism.

\section{Clinical risk factors for TE}

In univariate analysis, combination ICI therapy, Khorana Score $\geq 1$, and anticoagulation with or without prior history of DVT at treatment start were significantly associated with VTE $(\mathrm{p}<0.05$ for all $)$ (table 3$)$. Age $(\mathrm{p}=0.77)$, gender $(p=0.53)$, history of VTE $(p=0.77)$, history of CAD $(\mathrm{p}=0.06)$, the presence of brain metastases $(\mathrm{p}=0.08)$, and type of immunotherapy $(\mathrm{p}=0.07)$ were not significantly associated with VTE. In multivariate analysis, combination immunotherapy ((HR): 2.70; $95 \%$ CI: 1.28 to 5.70 ; $\mathrm{p}=0.009$ ), Khorana Score $\geq 1$ (HR: 2.24; 95\% CI: 1.06 to $4.74 ; \mathrm{p}=0.03$ ), and anticoagulation at treatment start (HR: $4.14 ; 95 \% \mathrm{CI}: 1.60$ to $10.7 ; \mathrm{p}=0.003$ ) remained significantly associated with VTE. History of CAD was also significantly associated with VTE in multivariate analysis (HR: 2.71; 95\% CI: 1.16 to 6.29 ; $\mathrm{p}=0.02$ ). To interrogate VTE prior to treatment initiation as a risk factor for subsequent incidence, we performed additional analysis excluding the eight patients on anticoagulation for DVT prior to treatment. Similar results were observed for combination immunotherapy $(\mathrm{p}=0.04)$, Khorana Score $\geq 1(p=0.008)$ and history of CAD $(p=0.07)$. In univariate analysis, no clinical factors were significantly associated with ATE.

\section{TE and association with OS}

The median OS for patients with TE was 20.0 months versus not reached for those without TE (HR: 2.33; 95\% CI: 1.40 to $3.88 ; \mathrm{p}<0.001)$. Due to the interaction of brain metastases with TE, we conducted separate analyses of the association of TE with survival in patients with and without brain metastasis. Patients with brain metastases $(n=43)$ had poor OS, regardless of TE status (2-year OS of $37.0 \%$ in patients without $\mathrm{TE}$ vs $31.3 \%$ in patients with $\mathrm{TE} ; \mathrm{p}$ value $=0.96$ ). Among patients without brain metastases $(n=185)$, OS was worse in patients with TE compared with those without TE (2-year OS of $50.8 \%$ vs $71.3 \%$; HR: 2.27 ; $95 \%$ CI: 1.36 to 3.79 ; $p=0.002$ ), when adjusted for age and stage in multivariate analysis (figure 3). Clinical variables associated with survival in univariate analysis included TE, age $>65$ years, and stage IV disease $(\mathrm{p}<0.001$ for all). Clinical variables associated with survival in multivariable analysis included TE (HR: 2.27; 95\% CI: 1.36 to 3.79; $\mathrm{p}=0.002$ ), age $>65$ years (HR: 1.97 ; $95 \%$ CI: 1.36 to $3.60 ; \mathrm{p}<0.001$ ) and stage IV disease (HR: 12.16; 95\% CI: 2.98 to 49.64; $\mathrm{p}<0.001$ ) (table 4). Gender $(\mathrm{p}=0.91)$, history of VTE $(\mathrm{p}=0.37)$, history of ATE $(\mathrm{p}=0.68)$, history of $\mathrm{CAD}(\mathrm{p}=0.58)$, anticoagulation at treatment start $(\mathrm{p}=0.29)$, and Khorana Score $\geq 1$ were not significantly associated with OS ( $p=0.26$ ). 
A: Without Brain Metastases

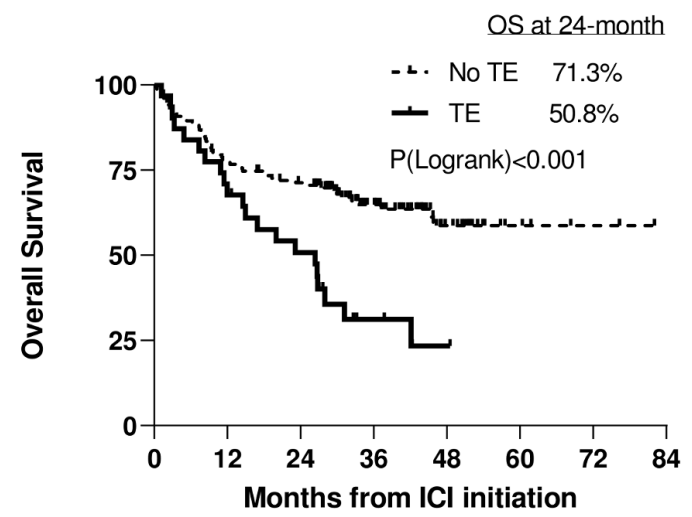

B: With Brain Metastases

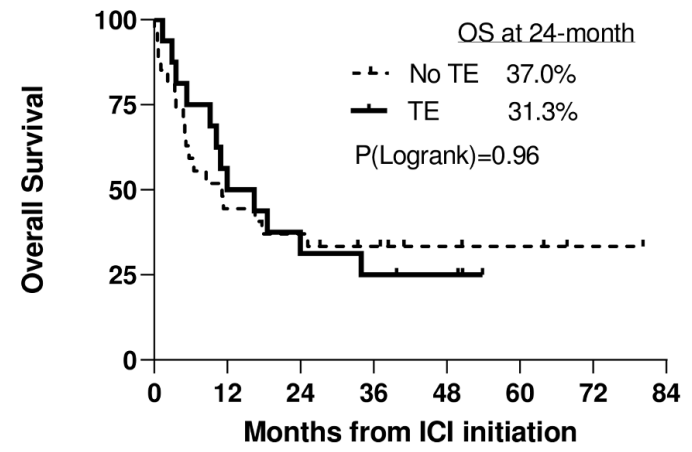

Figure 3 Kaplan-Meier curves for OS of melanoma patients after $\mathrm{ICl}$ initiation. (A) OS in patients without brain metastases $(n=185)$ stratified by the presence or the absence of TE $(p<0.001)$. (B) OS in patients with brain metastases $(n=43)$ stratified by the presence or the absence of TE $(p=0.96)$. $\mathrm{ICI}$, immune checkpoint inhibitor; OS, overall survival; TE, thromboembolism.

\section{DISCUSSION}

To the best our knowledge, we report the first clinical characterization of the incidence of TE and its association with survival and other outcomes in patients with melanoma receiving ICI. We found a substantial incidence of VTE and ATE after initiation of ICI in patients with melanoma. Additionally, we found that TE contributes to increased hospitalizations, treatment delays or discontinuation, and adversely impacts OS.
In this study, we report higher rates of TE in patients with melanoma during the era of ICI therapy, when compared with conventional chemotherapy (6-month cumulative incidence rate of $9.3 \%$ vs $2.6 \%-4.67 \%) .{ }^{6}{ }^{12}$ However, limited data are available for patients with melanoma in the era of chemotherapy when compared with other cancer types, most likely due to decreased OS ( 9 months). ${ }^{21}$ Rates of TE in our study are comparable to rates reported in other cancer types receiving ICI, which range from $2.6 \%$ to $5.2 \% .{ }^{1422}$ Limited data are available for ATE. A large pharmacovigilance study demonstrated increased association of reporting cardiovascular events, like myocarditis, pericardial disease, and vasculitis with ICI, when compared with any drugs. ${ }^{23}$ A case report and a case series demonstrated arterial thrombi in iliac arteries, subclavian artery, and peripheral arteries in patients with melanoma treated with ICI. ${ }^{24} 25$ A meta-analysis of 22 clinical trials to evaluate immune-related adverse events in non-small cell lung cancer revealed ATE incidence rate of $4 \%$ (including cardiac arrest, MI, and stroke), which is similar to our study. ${ }^{26}$ Additionally, a pooled analysis of cardiovascular adverse events in ICI trials demonstrated an incidence rate of $8.93 \%$ for vascular/thromboembolic/bleeding events, $0.30 \%$ sudden cardiac death, and $0.58 \%$ ischemic events. Incidence rates were higher in patients receiving combination ICI: $11.6 \%$ versus $8.69 \%$ vascular/thromboembolic/bleeding, $0.46 \%$ versus $0.28 \%$ sudden cardiac death, and $1.3 \%$ versus $0.51 \%$ ischemic events. ${ }^{27}$ These findings are consistent with our study, as higher rates of VTE were observed with combination ICI compared with ICI monotherapy. While not statistically significant, we observed numerically higher rates of ATE with combination therapy compared with ICI monotherapy ( $5.1 \%$ vs $3 \%$ at 12 months).

The mechanism for cancer-associated thrombosis in the setting of ICI use is not entirely understood. Several studies have demonstrated that cancer cells are capable of directly activating the coagulation cascade by increasing expression of tissue factor and plasminogen activator inhibitor-1, and release of microparticles, among activation of other coagulation factors. ${ }^{28-31}$ Additionally, cancer cells indirectly activate the coagulation cascade by synthesizing and secreting inflammatory cytokines, and also stimulate host-cell production of inflammatory cytokines like tumor necrosis factor(TNF)-alpha

Table 4 Multivariate analysis for associations between clinical factors and OS in patients without brain metastases

\begin{tabular}{|c|c|c|c|c|c|c|c|}
\hline Variable & Factor & $\mathrm{N}=185$ & VTE no. (\%) & 2-year OS $(95 \% \mathrm{Cl})$ & HR & $95 \% \mathrm{Cl}$ & $P$ value \\
\hline \multirow[t]{2}{*}{ TE after ICI } & No & 154 & $53(34)$ & 71.3 (64.0 to 78.5$)$ & & & \\
\hline & Yes & 31 & $21(68)$ & 50.8 (33.0 to 68.6$)$ & 2.27 & 1.36 to 3.79 & 0.002 \\
\hline Age at treatment start, years & $>65$ & 97 & $49(51)$ & 60.2 (50.2 to 70.2$)$ & 2.22 & 1.36 to 3.60 & $<0.001$ \\
\hline Stage at treatment start & III & 43 & $2(5)$ & $100.0(100.0$ to 100.0$)$ & & & \\
\hline
\end{tabular}

$\mathrm{ICI}$, immune checkpoint inhibitor; OS, overall survival; TE, thromboembolism; VTE, venous thromboembolism. 
and interleukin-1 $\beta^{32}{ }^{33}$ This already pro-inflammatory state may be further exacerbated with programmed cell death protein-1 (PD-1) and/or cytotoxic T-lymphocyteassociated protein-4 (CTLA-4) inhibition. Production of inflammatory cytokines subsequently suppresses the antithrombotic response of endothelial cells and promotes procoagulant activity. ${ }^{34}$ Recent studies have suggested that aberrant activation of the innate immune response is associated with thrombosis and atherothrombosis. ${ }^{35}$

Additionally, several studies have investigated the role of PD-1 and its impact on atherogenesis. PD-1 has a critical role in downregulating proatherogenic $\mathrm{T}$ cell responses, and blockade of PD-1 has been shown in murine models to accelerate atherogenesis with findings of increased infiltration of macrophages, $\mathrm{CD} 4+$ and $\mathrm{CD} 8+\mathrm{T}$ cells in aortic plaques. ${ }^{36}$ These preclinical models suggest that PD-1 inhibitors can increase the risk of cardiovascular complications, like ATE.

Our findings also suggest worse clot-related morbidity and mortality in patients with TE, which is consistent with prior studies in patients with cancer. A significant proportion of patients (72\%) were hospitalized for TE, and 19\% resulted in clot-related mortality. Of patients without brain metastases, those with TE had twofold worse survival than patients without TE. A potential explanation for the observed worsened survival in patients on ICI therapy who developed ATE may be due to reprogramming of the innate immune system to accelerate cancer growth. A recent preclinical study in breast cancer demonstrated that MI causes reprogramming of monocytes in the bone marrow to an immunosuppressive phenotype that was maintained in circulation and recruited to the tumor, resulting in accelerated breast cancer growth. ${ }^{38}$ Similarly, another study has demonstrated that activation of coagulation by tissue factor-thrombin-protease-activated receptor-1 signaling in pancreatic cancer alters the immune regulation pathway by suppressing CD8+ T cells in the tumor microenvironment, and potentially evading the immune system. ${ }^{39}$ Analysis of multiple cancer cell types, including melanoma, has demonstrated that increased activity of tissue factor, thrombin, or prothrombin increases metastatic disease burden. ${ }^{40}$ Cancer associated thrombosis can suppress the immune system's response to cancer and subsequently lead to a worse prognosis. These findings have implications for the use of ICI therapy in melanoma and other malignancies.

Our study has several limitations. First, our study cohort comprised retrospective data derived from a single health system. However, the sample size and detailed patient follow-up enabled by the use of a single electronic medical record system coupled with manual chart review allowed us to capture VTE and ATE events. Another limitation is that other risk factors for thrombosis like smoking and hypertension were not collected. Smoking is more commonly associated with other cancers, like lung and genitourinary cancers, as compared with melanoma, and accuracy in the electronic medical record for these risk factors is limited. ${ }^{41-44}$ Additionally, the variable of anticoagulation in patients with or without prior DVT at treatment start represents anticoagulants that were prescribed by providers to patients at the time of therapy initiation. However, it remains unclear if patients were taking the medication during ICI therapy, as this was not documented in provider notes. Anticoagulation at treatment initiation may also be an indicator of underlying comorbidities that were not adequately captured in this cohort study.

These results have significant implications for patient care. Recent prospective clinical trials demonstrate that VTE in high-risk patients with cancer (defined as Khorana Score $\geq 2$ ) treated with conventional chemotherapy can be prevented with prophylactic anticoagulation and reducing rate of VTE by up to $6 \%{ }^{45}{ }^{46}$ Thromboprophylaxis with apixaban, rivaroxaban, or low molecular weight heparin is indicated for high-risk outpatients with cancer prior to starting a new systemic chemotherapy regimen. ${ }^{18}$ If our results are confirmed by others, thromboprophylaxis could be considered in our patient population receiving ICI, although notably anticoagulation did not appear to be protective in our cohort of patients with a prior history of VTE. Additionally, the safety and efficacy of anticoagulation in patients with melanoma treated with ICI, some of whom have vascular brain metastases, and can develop immunemediated colitis, is unknown, and additional prospective studies are needed. A recent retrospective study evaluating melanoma patients with brain metastases and VTE $(n=74)$ demonstrated that of 57 patients $(77 \%)$ who received anticoagulation, 2 (4\%) developed intracranial hemorrhage, which was not statistically different from those who did not receive anticoagulation. ${ }^{47}$ Additionally, OS in patients with VTE who received anticoagulation was longer (median OS: 4.2 months vs 1.2 months, $\mathrm{p}=0.06) .{ }^{47}$ These data suggest potential safety and improved OS in melanoma patients receiving therapeutic anticoagulation for VTE. For now, clinicians and patients should be aware of the high risk of VTE and ATE, and the implications for prognosis once such events have occurred.

Contributors TAS collected data, analyzed data, and wrote manuscript. HL analyzed data. BH interpreted data and reviewed manuscript. KRM interpreted data and reviewed manuscript. PF reviewed manuscript. AAK designed the research, analyzed data, and revised the manuscript.

Funding The authors have not declared a specific grant for this research from any funding agency in the public, commercial or not-for-profit sectors.

Competing interests PF receives institutional research funding from Pfizer and Bristol Myers Squibb, outside the submitted work. BH reports research funds from Scientific Advisory for Bayer AG, Presagia, and STCube Pharmaceuticals, outside the submitted work. AAK reports personal fees and non-financial support from Janssen, Bayer, Sanofi, Halozyme, Seattle Genetics, Pfizer, and Medscape, personal fees from Parexel and TriSalus, grants from Merck, Array, and Leap, and grants and personal fees from Bristol Myers Squibb, outside the submitted work.

Patient consent for publication Not required.

Ethics approval Study was approved by the IRB at the Cleveland Clinic.

Provenance and peer review Not commissioned; externally peer reviewed.

Data availability statement All data relevant to the study are included in the article or uploaded as supplementary information. N/A.

Open access This is an open access article distributed in accordance with the Creative Commons Attribution Non Commercial (CC BY-NC 4.0) license, which 
permits others to distribute, remix, adapt, build upon this work non-commercially, and license their derivative works on different terms, provided the original work is properly cited, appropriate credit is given, any changes made indicated, and the use is non-commercial. See http://creativecommons.org/licenses/by-nc/4.0/.

\section{ORCID iD}

Tamara A Sussman http://orcid.org/0000-0003-3429-4468

\section{REFERENCES}

1 Khorana AA, Francis CW, Culakova E, et al. Frequency, risk factors, and trends for venous thromboembolism among hospitalized cancer patients. Cancer 2007;110:2339-46.

2 Blom JW, Doggen CJM, Osanto S, et al. Malignancies, prothrombotic mutations, and the risk of venous thrombosis. JAMA 2005;293:715-22.

3 Sørensen HT, Mellemkjaer L, Olsen JH, et al. Prognosis of cancers associated with venous thromboembolism. N Engl J Med 2000;343:1846-50.

4 Lyman GH, Eckert L, Wang Y, et al. Venous thromboembolism risk in patients with cancer receiving chemotherapy: a real-world analysis. Oncologist 2013;18:1321-9.

5 Khorana AA, Francis CW, Culakova E, et al. Thromboembolism is a leading cause of death in cancer patients receiving outpatient chemotherapy. J Thromb Haemost 2007;5:632-4.

6 Navi BB, Reiner AS, Kamel H, et al. Risk of Arterial Thromboembolism in Patients With Cancer. J Am Coll Cardio 2017;70:926-38.

7 Khorana AA, Kuderer NM, Culakova E, et al. Development and validation of a predictive model for chemotherapy-associated thrombosis. Blood 2008;111:4902-7.

8 Ay C, Dunkler D, Marosi C, et al. Prediction of venous thromboembolism in cancer patients. Blood 2010;116:5377-82.

9 Thaler J, Ay C, Pabinger I. Venous thromboembolism in cancer patients - risk scores and recent randomised controlled trials. Thromb Haemost 2012;108:1042-8.

10 Gervaso L, Montero AJ, Jia X, et al. Venous thromboembolism in breast cancer patients receiving cyclin-dependent kinase inhibitors. $J$ Thromb Haemost 2020;18:162-8.

11 Petrelli F, Cabiddu M, Borgonovo K, et al. Risk of venous and arterial thromboembolic events associated with anti-EGFR agents: a metaanalysis of randomized clinical trials. Ann Oncol 2012;23:1672-9.

12 Marks MA, Engels EA. Venous thromboembolism and cancer risk among elderly adults in the United States. Cancer Epidemiol Biomarkers Prev 2014:23:774-83.

13 Sparsa A, Durox H, Doffoel-Hantz V, et al. High prevalence and risk factors of thromboembolism in stage IV melanoma. J Eur Acad Dermatol Venereol 2011;25:340-4.

14 Ando Y, Hayashi T, Sugimoto R, et al. Risk factors for cancerassociated thrombosis in patients undergoing treatment with immune checkpoint inhibitors. Invest New Drugs 2020;38:1200-6.

15 Wolchok JD, Chiarion-Sileni V, Gonzalez R, et al. Overall survival with combined nivolumab and ipilimumab in advanced melanoma. N Engl J Med 2017;377:1345-56.

16 Khorana AA, Francis CW, Kuderer NM, et al. Dalteparin thromboprophylaxis in cancer patients at high risk for venous thromboembolism: a randomized trial. Thromb Res 2017;151:89-95.

17 Streiff MB, Bockenstedt PL, Cataland SR, et al. Venous thromboembolic disease. J Natl Compr Canc Netw 2013;11:1402-29.

18 Key NS, Khorana AA, Kuderer NM, et al. Venous thromboembolism prophylaxis and treatment in patients with cancer: ASCO clinical practice guideline update. J Clin Oncol 2020;38:496-520.

19 Fine JP, Gray RJ. A proportional hazards model for the Subdistribution of a competing risk. J Am Stat Assoc 1999;94:496-509.

20 Akaike $\mathrm{H}$. A new look at the statistical model identification. IEEE Trans Automat Contr 1974;19:716-23.

21 Patel PM, Suciu S, Mortier L, et al. Extended schedule, escalated dose temozolomide versus dacarbazine in stage IV melanoma: final results of a randomised phase III study (EORTC 18032). Eur J Cancer 2011;47:1476-83.

22 Bar J, Markel G, Gottfried T, et al. Acute vascular events as a possibly related adverse event of immunotherapy: a single-institute retrospective study. Eur J Cancer 2019;120:122-31.

23 Salem J-E, Manouchehri A, Moey M, et al. Cardiovascular toxicities associated with immune checkpoint inhibitors: an observational, retrospective, pharmacovigilance study. Lancet Oncol 2018;19:1579-89.

24 Thoreau B, Gouaillier-Vulcain F, Machet L, et al. Acute lower limb ischaemia and diabetes in a patient treated with anti-PD1 monoclonal antibody for metastatic melanoma. Acta Derm Venereol 2017;97:408-9.

25 Boutros C, Scoazec J-Y, Mateus C, et al. Arterial thrombosis and anti-PD-1 blockade. Eur J Cancer 2018;91:164-6.

$26 \mathrm{Hu}$ Y-B, Zhang Q, Li H-J, et al. Evaluation of rare but severe immune related adverse effects in PD-1 and PD-L1 inhibitors in non-small cell lung cancer: a meta-analysis. Transl Lung Cancer Res 2017;6:S8-20.

27 Amiri-Kordestani L, Moslehi J, Cheng J, et al. Cardiovascular adverse events in immune checkpoint inhibitor clinical trials: a U.S. food and drug administration pooled analysis. JCO 2018;36:3009

28 Khorana AA, Ahrendt SA, Ryan CK, et al. Tissue factor expression, angiogenesis, and thrombosis in pancreatic cancer. Clin Cancer Res 2007;13:2870-5.

29 Andrén-Sandberg A, Lecander I, Martinsson G, et al. Peaks in plasma plasminogen activator inhibitor-1 concentration may explain thrombotic events in cases of pancreatic carcinoma. Cancer 1992;69:2884-7

30 Lupu-Meiri M, Geras-Raaka E, Lupu R, et al. Knock-Down of plasminogen-activator inhibitor-1 enhances expression of Ecadherin and promotes epithelial differentiation of human pancreatic adenocarcinoma cells. J Cell Physiol 2012;227:3621-8.

31 Muhsin-Sharafaldine M-R, Saunderson SC, Dunn AC, et al. Procoagulant and immunogenic properties of melanoma exosomes, microvesicles and apoptotic vesicles. Oncotarget 2016;7:56279-94.

32 Bevilacqua MP, Pober JS, Majeau GR, et al. Recombinant tumor necrosis factor induces procoagulant activity in cultured human vascular endothelium: characterization and comparison with the actions of interleukin 1. Proc Natl Acad Sci U S A 1986;83:4533-7.

33 Falanga A, Panova-Noeva M, Russo L. Procoagulant mechanisms in tumour cells. Best Pract Res Clin Haematol 2009;22:49-60.

34 Sato R, Imamura K, Sakata S, et al. Disorder of coagulationfibrinolysis system: an emerging toxicity of anti-PD-1/PD-L1 monoclonal antibodies. J Clin Med 2019;8:762.

35 Gaertner F, Massberg S. Blood coagulation in immunothrombosisAt the frontline of intravascular immunity. Semin Immunol 2016;28:561-9.

36 Cochain C, Chaudhari SM, Koch M, et al. Programmed cell deathdeficiency exacerbates $T$ cell activation and atherogenesis despite expansion of regulatory T cells in atherosclerosis-prone mice. PLoS One 2014;9:e93280.

37 Bu D-xiu, Tarrio M, Maganto-Garcia E, et al. Impairment of the programmed cell death-1 pathway increases atherosclerotic lesion development and inflammation. Arterioscler Thromb Vasc Biol 2011;31:1100-7.

38 Koelwyn GJ, Newman AAC, Afonso MS, et al. Myocardial infarction accelerates breast cancer via innate immune reprogramming. Nat Med 2020;26:1452-8.

39 Yang Y, Stang A, Schweickert PG, et al. Thrombin signaling promotes pancreatic adenocarcinoma through PAR-1-Dependent immune evasion. Cancer Res 2019;79:3417-30.

40 Mueller BM, Reisfeld RA, Edgington TS, et al. Expression of tissue factor by melanoma cells promotes efficient hematogenous metastasis. Proc Natl Acad Sci U S A 1992;89:11832-6.

41 Thun MJ, Lally CA, Flannery JT, et al. Cigarette smoking and changes in the histopathology of lung cancer. J Natl Cancer Inst 1997;89:1580-6.

42 Freedman ND, Silverman DT, Hollenbeck AR, et al. Association between smoking and risk of bladder cancer among men and women. JAMA 2011;306:737-45.

43 Song F, Qureshi AA, Gao X, et al. Smoking and risk of skin cancer: a prospective analysis and a meta-analysis. Int $\mathrm{J}$ Epidemiol 2012;41:1694-705

44 DeLancey JO, Hannan LM, Gapstur SM, et al. Cigarette smoking and the risk of incident and fatal melanoma in a large prospective cohort study. Cancer Causes Control 2011;22:937-42.

45 Khorana AA, Soff GA, Kakkar AK, et al. Rivaroxaban for thromboprophylaxis in high-risk ambulatory patients with cancer. $N$ Engl J Med 2019;380:720-8.

46 Carrier M, Abou-Nassar K, Mallick R, et al. Apixaban to prevent venous thromboembolism in patients with cancer. $N$ Engl J Med 2019;380:711-9.

47 Alvarado G, Noor R, Bassett R, et al. Risk of intracranial hemorrhage with anticoagulation therapy in melanoma patients with brain metastases. Melanoma Res 2012;22:310-5. 